\title{
Resultados de aprendizagem no quadro do ensino superior português: $O$ caso da psicologia e da educação
}

\section{Measuring learning outcomes in Portuguese Higher Education:}

\author{
An analysis of Psychology and Education syllabus
}

\author{
Diana Soares*, Diana Dias* \\ * Universidade Europeia
}

\begin{abstract}
Resumen
A complexidade inerente à definição e construção dos resultados de aprendizagem pelas instituições de ensino superior tornam este conceito alvo de múltiplas leituras e entendimentos. Partindo desta diversidade de perspetivas, este estudo centra-se na análise semântica dos resultados de aprendizagem propostos nas áreas científicas de psicologia e de educação. Recorrendo a uma metodologia qualitativa, foram analisadas, qualitativa $\mathrm{e}$ semanticamente, as respostas à questão "resultados de aprendizagem a adquirir pelos estudantes" dos Pedidos de Acreditação de Ciclos de Estudo submetidos à A3ES. Os resultados apontam para diferentes conhecimentos específicos e competências transversais em função dos ciclos de estudo. Algumas convergências entre as duas áreas científicas são também encontradas. Estes dados possibilitam um melhor conhecimento dos resultados de aprendizagem preconizados pelas IES português enquanto perfil de competências associado aos cursos de psicologia e educação.,

Palabras clave: resultados de aprendizagem, conhecimentos, competências, educação e psicología.
\end{abstract}

\footnotetext{
Abstract

The complexity inherent to the definition and elaboration of learning outcomes by higher education institutions makes this concept ambiguous and nebulous. Based on this diversity of perspectives, this study focuses on the semantic analysis of the proposed learning outcomes in the scientific areas of psychology and education. Using a qualitative methodology, the answers from the question "learning outcomes to be acquired by the students" were analyzed qualitatively and semantically, in the scope of the protocols submitted

to the A3ES. The results reveal different specific knowledge and transversal competences depending on the study cycles. Some convergences between the two scientific areas are also found. These data allow a better knowledge of the learning outcomes advocated by the Portuguese HEI as a profile of competences associated to the psychology and education courses.

Keywords: learning outcomes, competences, education and psychology
}

\section{Introdução}

Como resposta aos desafios colocados pela Declaração de Bolonha, o sistema de ensino superior português (e europeu) tem vindo a transformar significativamente, não só, as suas políticas como também a sua praxis (Dias \& Amaral, 2014). A necessidade de construir sistemas de educação comparáveis, compatíveis e, de certa forma, transferíveis entre si, tem originado alterações na forma como os conteúdos e os respetivos programas curriculares são desenhados e concebidos. É esperado, por exemplo, que as metodologias de avaliação, os processos de ensino-aprendizagem, os objetivos curriculares e os resultados de aprendizagem (RA learning outcomes) estejam alinhados entre si, permitindo a almejada comparabilidade e transparência entre os sistemas educativos (Veiga, Rosa, Dias, \& Amaral, 2013). Assim, cada nível de qualificação (cada ciclo de estudo) deve ser definido em termos do que se espera que o estudante conheça, compreenda e seja capaz de desempenhar no final dessa formação (Dias \& Amaral, 2014). Ou seja, cada qualificação integra um conjunto específico de RA, que acaba por corresponder ao perfil de competências técnicas e/transversais esperadas para aquela formação/ciclo de estudos (Dias \& Soares, 2017). Usando os RA enquanto medida de análise, a ênfase passa a ser colocada no que se espera que um estudante adquira em termos de conhecimento, habilidades e competências, em função de cada nível de qualificação (Gallavara, Hreinsson, Kajaste, \& Zadeh, 2008; Tissot, 2008). Além disso, os RA devem explicitar, não só o que se espera que os estudantes venham a saber, como também os critérios e indicadores requeridos para a sua avaliação e monitorização (Adam, 2004; Moon, 2004; Wiliam, 2010).

$O$ conceito de RA reveste-se, em si mesmo, de alguma controvérsia e ambiguidade (Aamodt \& Hovdhaugen, 2008; Adam, 2008; Scott, 2011). Na verdade, uma grande diversidade de definições e perspetivas sobre este conceito emergem na literatura, sob a forma de múltiplas taxonomias e tipologias de 
conhecimentos, competências, aptidões e valores. Por exemplo, no estudo internacional Assessment of Higher Education Learning Outcomes - AHELO (Tremblay, Lalancette, Roseveare, Dias, \& Amaral, 2013), os RA foram definidos enquanto a combinação de habilidades genéricas e habilidades específicas inerentes à área científica. $O$ pensamento crítico, o conhecimento disciplinar, a resolução de problemas, o trabalho em equipa, a comunicação, as habilidades profissionais, a ética e os valores cívicos, a criatividade e a aprendizagem ao longo da vida são exemplos das dimensões mais significativas de RA. No projeto TUNNING (Gonzalez \& Wagenaar, 2003), diferenciaram-se três tipos de competências genéricas: i) as competências instrumentais (habilidades cognitivas, habilidades metodológicas, habilidades tecnológicas e habilidades linguísticas); ii) as competências interpessoais (habilidades sociais, de interação social e cooperação); e as iii) as competências sistêmicas (habilidades mais complexas, como a combinação de compreensão, sensibilidade e conhecimento, necessária aquisição prévia de competências instrumentais e interpessoais). Desta taxonomia fazem parte trinta e uma competências genéricas, relacionadas, por exemplo, com a capacidade de comunicar num segundo idioma, a capacidade de aprender ao longo da vida, a capacidade de comunicação oral e escrita, a capacidade de autoavaliação, a capacidade de planear e gerir o tempo, a capacidade de gerar novas ideias, a capacidade de pesquisar, processar e analisar informação, a capacidade de identificar, colocar e resolver problemas, a capacidade de tomar decisões, a capacidade de realizar pesquisas, a capacidade de trabalhar em equipa ou em contextos internacionais, a capacidade de atuar com base em princípios éticos e deontológicos, entre outras. Para Nusche (2008), os RA podem ser de base cognitiva (conhecimentos mais específicos e em processos de pensamento e de resolução de problemas). Estes RA de base cognitiva incluem a aquisição de conhecimentos genéricos e específicos, bem como a aquisição de determinadas habilidades/aptidões, tais como o raciocínio verbal e numérico, o processamento analítico, o pensamento crítico ou a resolução de problemas. Estas habilidades são já transferíveis a diferentes áreas disciplinares e contextos de aprendizagem. Por outro lado, as habilidades não cognitivas referem-se a mudanças nas crenças ou à evolução de certos padrões e valores cívicos (Ewell, 2005). O desenvolvimento psicossocial engloba o desenvolvimento pessoal, nomeadamente o desenvolvimento da identidade e da autoestima, bem como a criação de relações interpessoais. A consciencialização social, a motivação para aprender e o respeito pela diversidade cultural são também exemplos de RA de cariz não cognitivos (Reason \& Hemer, 2012).

Partindo da definição de RA e das múltiplas taxonomias apresentadas, este estudo pretende analisar que conhecimentos e competências são valorizadas enquanto RA dos cursos de Psicologia e Educação, procurando pontos de convergência e divergência nos perfis dos graduados destas duas áreas científicas.

\section{Metodologia}

\section{Medidas}

$\mathrm{Na}$ fase inicial do processo de avaliação e acreditação dos ciclos de estudos (novos ou em funcionamento), as Instituições de Ensino Superior necessitam submeter um pedido de acreditação à Agência de Avaliação e Acreditação do Ensino Superior (A3ES). Nesse formulário, uma das questões solicita a descrição dos resultados de aprendizagem a adquirir pelos estudantes no decurso dessa qualificação, descrição essa que se encontra circunscrita a 1000 caracteres. A análise foca-se nas respostas dadas especificamente a essa questão, tentando sistematizar o conteúdo das mesmas, de forma a extrair significado a partir de texto não tratado. Tendo em conta o objetivo deste estudo, a análise circunscreveu-se às áreas científicas de Psicologia (CNAEF 311) e Ciências da Educação (CNAEF 142). Foram analisados todos os pedidos de acreditação de ciclos de estudo destas duas áreas científicas, submetidos à A3ES desde 2009 até 2015. No total, foram analisados 102 ciclos de estudos de Psicologia e 47 de Ciências da Educação.

\section{Procedimentos}

Seguindo uma abordagem qualitativa, foi realizada uma análise de conteúdo aos resultados de aprendizagem apresentados nos pedidos de acreditação de Novos Ciclos de Estudo e Ciclos de Estudo em Funcionamento, submetidos à A3ES.

A análise de conteúdo foi elaborada partindo de uma matriz de análise, previamente elaborada para o efeito, já validada em termos teóricos e empíricos (Dias \& Soares, 2017). Esta matriz subdivide-se em competências específicas (conhecimentos gerais, específicos, práticos e teóricos) e competências transversais (tais como, pensamento crítico, inovação, motivação para excelência, resolução de problemas, comunicação ou valores éticos e deontológicos). Neste sentido, este procedimento de análise encontra-se enquadrado mais numa lógica de uma theoretical thematic analysis em vez de uma data-driven approach (Braun \& Clarke, 2006). A análise pretendeu, assim, encontrar padrões/temas que se enquadrassem dentro da taxonomia de competências técnicas e transversais da matriz da analise, permitindo transformar o conteúdo dos RA em unidades de texto associadas a competências.

\section{Resultados}

Tendo por base a matriz de análise, a análise de conteúdo efetuada permite perceber que competências são mais referenciadas nos ciclos de estudos de Psicologia e de Ciências da Educação. Na tabela 1, apresentam-se as percentagens de cada uma das competências (específicas e transversais) para o total de amostra e contrastando ambas as áreas científicas. Estas percentagens são relativas, considerando já o número total de documentos por cada área científica. 
Tabela 1.

Frequências relativas de competências especificas e transversais por área cientifica

\begin{tabular}{|c|c|c|c|}
\hline Categorias & Ciências da Educação & Psicologia & Total \\
\hline Conhecimentos gerais & 55,81 & 8,82 & 22,76 \\
\hline Conhecimentos específicos & 53,49 & 77,45 & 70,34 \\
\hline a) Conhecimentos práticos & 9,30 & 47,06 & 35,86 \\
\hline b) Conhecimentos teóricos & 2,33 & 38,24 & 27,59 \\
\hline Tomada de decisão & 9,30 & 7,84 & 8,28 \\
\hline Resolução de problemas & 23,26 & 20,59 & 21,38 \\
\hline Capacidade de organização e planeamento & 60,47 & 6,86 & 22,76 \\
\hline Análise crítica e reflexiva/pensamento crítico & 65,12 & 64,71 & 64,83 \\
\hline Criatividade & 23,26 & 6,86 & 11,72 \\
\hline Capacidade de comunicação oral & 25,58 & 40,20 & 35,86 \\
\hline Capacidade de comunicação escrita & 25,58 & 37,25 & 33,79 \\
\hline Domínio de uma língua estrangeira & 0,00 & 1,96 & 1,38 \\
\hline Capacidade de gestão da informação & 2,33 & 17,65 & 13,10 \\
\hline Domínio das tecnologias da informação & 9,30 & 0,98 & 3,45 \\
\hline Trabalho em equipa & 20,93 & 19,61 & 20,00 \\
\hline a) Trabalho em equipas interdisciplinares & 2,33 & 18,63 & 13,79 \\
\hline b) Trabalho em equipa internacional & 2,33 & 3,92 & 3,45 \\
\hline Capacidade de relacionamento interpessoal & 13,95 & 8,82 & 10,34 \\
\hline a) Capacidade de negociação & 0,00 & 3,92 & 2,76 \\
\hline b) Gestão de conflitos & 0,00 & 1,96 & 1,38 \\
\hline Motivação para a excelência & 9,30 & 18,63 & 15,86 \\
\hline Capacidade de gestão da informação & 11,63 & 0,00 & 3,45 \\
\hline Adaptação a novas situações & 4,65 & 25,49 & 19,31 \\
\hline Iniciativa e espírito empreendedor & 11,63 & 5,88 & 7,59 \\
\hline Inovação & 25,58 & 12,75 & 16,55 \\
\hline Motivação aprendizagem contínua/longo vida & 30,23 & 20,59 & 23,45 \\
\hline Liderança & 9,30 & 2,94 & 4,83 \\
\hline Desenvolvimento de trabalho autónomo & 30,23 & 29,41 & 29,66 \\
\hline Preocupação com a sustentabilidade social, eco & 4,65 & 3,92 & 4,14 \\
\hline Responsabilidade pessoal e social & 25,58 & 20,59 & 22,07 \\
\hline Ética e deontologia profissional & 20,93 & 36,27 & 31,72 \\
\hline Respeito pela diversidade e multiculturalidade & 16,28 & 3,92 & 7,59 \\
\hline
\end{tabular}

Considerando a totalidade dos documentos analisados (não diferenciando as áreas científicas), verifica-se os conhecimentos específicos correspondem à categoria com um maior número de referências, a par da análise crítica e reflexiva. Por outro lado, o domínio de uma língua estrangeira e a gestão de conflitos surgem como as categorias mais negligenciada nestas áreas científicas, com poucas referências. Destacam-se, assim, como competências técnicas, os conhecimentos específicos, tanto práticos como teóricos. Dentro das competências transversais, a ênfase é colocada na análise crítica e reflexiva, na capacidade de comunicação oral e escrita, na ética e na deontologia profissional e no desenvolvimento de trabalho autónomo.

Ao considerar apenas os RA da área científica de Ciências da Educação, verifica-se que as competências transversais de análise crítica e reflexiva (65\%) e capacidade de organização e planeamento $(60 \%)$ são as mais valorizadas. Com valores próximos, mas mais baixos, surgem os conhecimentos gerais $(56 \%)$ e os específicos (53\%), já dentro da macro categoria de competências técnicas). De destacar ainda, o peso dado às competências relacionadas com a capacidade de trabalho autónomo e aprendizagem ao longo da vida (perto de 30\%). Por fim, e ainda acima de uma representatividade de $25 \%$, as competências de comunicação oral e escrita e a responsabilidade pessoal e social. Por sua vez, na área científica de Psicologia, destacam-se as os conhecimentos específicos (77\%), seguidas das competências transversais análise crítica e reflexiva $(65 \%)$. As competências de comunicação oral e escrita e a ética e deontologia profissional apresentam, igualmente, valores elevados (próximos de 40\%). De referir ainda a adaptação a novas situações e o desenvolvimento de trabalho autónomo, com valores acima de $25 \%$.

Em termos de contraste entre as duas áreas científicas (figuras 1 e 2), as diferenças encontram-se ao nível dos conhecimentos gerais, capacidade de organização e planeamento particularmente enfatizadas pelos cursos de ciências de educação. Por outro lado, os conhecimentos específicos (teóricos e práticos), a adaptação a novas situações, a ética e deontologia profissional e o trabalho em equipas interdisciplinas são competências com destaque positivo (e diferenciador) nos cursos de psicologia.

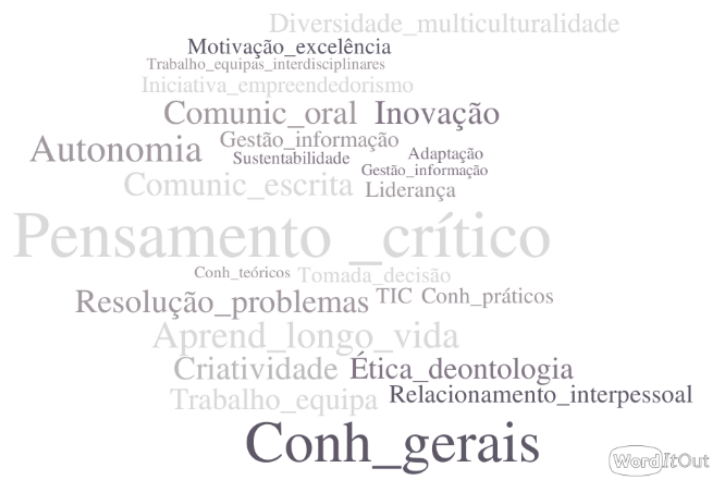

Fig. 1 Diagrama de palavras mais frequentes na área de Ciências da Educação

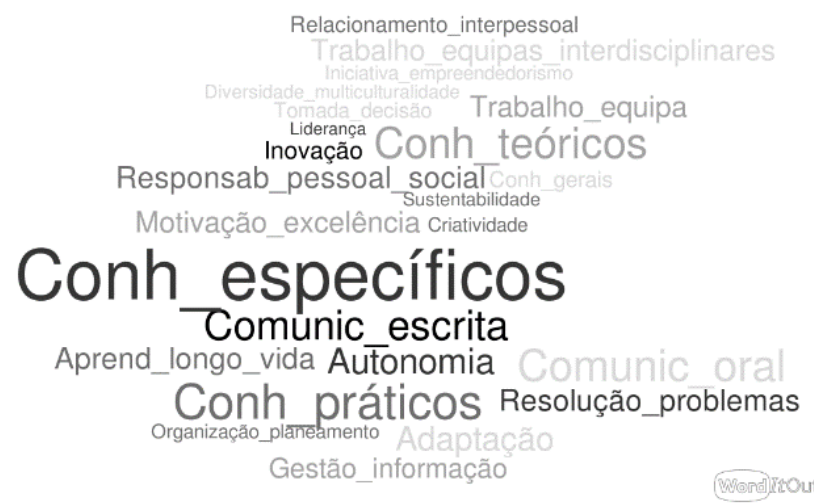

Fig.2 Diagrama de palavras mais frequentes na área de Psicologia

\section{Discussão e conclusão}

A análise de conteúdo aos RA propostos nos pedidos de acreditação de novos cursos ou cursos já em funcionamento das áreas da Psicologia e Ciências da Educação possibilitou compreender que conhecimentos e competências tendem a ser mais valorizadas pelas instituições de Ensino Superior, especificamente nestas áreas científicas. De acordo com os resultados, e 
considerando a totalidade dos documentos, verifica-se que tanto as competências mais específicas como as transversais assumem igual preponderância. Com efeito, os conhecimentos específicos e a análise crítica e reflexiva são as categorias mais referenciadas no total dos documentos. Os conhecimentos específicos (tanto teóricos como práticos) surgem como principal componente dos RA, sendo esperado, por exemplo, que os "estudantes adquiram conhecimentos ao nível das várias correntes teóricas da psicologia" (exemplo de RA de psicologia), ou que "detenham elevado conhecimento ao nível dos métodos e práticas pedagógicas a implementar em contexto de ensino básico" (exemplo de RA de Educação). Ao nível da competência transversal de análise crítica e reflexiva, outros estudos salientam a sua relevância, cada vez mais visível, ao nível do ensino superior (Soares, Franco, \& Dias, 2017). Esta competência, assumida como imprescindível no conjunto das competências do século XXI (Egan, Maguire, Christophers, \& Rooney, 2017), é valorizada não só no âmbito dos conteúdos curriculares, como também pelos próprios estudantes e docentes. Sendo, tradicionalmente, o ensino superior a última etapa de educação formal, neste contexto de transição do sistema educativo para o do emprego/trabalho, esta competência surge como principal soft-skill ou competência transversal.

Particularizando a área científica de psicologia, destacam-se conhecimentos específicos, enquanto competências mais técnicas, a par de um conjunto significativo de competências transversais, tais como a análise crítica e reflexiva, a comunicação oral e escrita e a ética e deontologia profissional. Importa salientar a relevância desta última competência, imprescindível à prática profissional do/a psicólogo/a em qualquer contexto de intervenção, tal como afirmado no código deontológico da Ordem dos Psicólogos (OPP, 2011). No caso das ciências da educação, as competências transversais assumem maior peso que os conhecimentos ou competências técnicas. A análise crítica e reflexiva e a capacidade de organização e planeamento são as categorias mais valorizadas. Dentro dos conhecimentos, destacam-se os conhecimentos gerais (seguidos dos específicos). Estes contrastes em termos de perfis de competências traduzem as próprias especificidades das áreas científicas. O perfil de competências de um graduado em ciências da educação é mais genérico e abrangente, possibilitando a sua atuação do profissional em diversos contextos de intervenção. Daí a relevância dos conhecimentos gerais/genéricos que, a par dos conhecimentos específicos, apoiam a sua adaptação a esta multiplicidade de contextos, problemas e situações profissionais. O perfil de competências do psicólogo tende já a ser mais circunscrito, incidindo fortemente nos conhecimentos específicos (tanto teóricos como práticos) e em competências de cariz ético e deontológico, tal como regulamentado pela sua ordem profissional. Também a adaptação a novas situações e o trabalho em equipas interdisciplinas surgem como categorias de referência nesta área científica, aproximando-se daquilo que serão os desafios profissionais e as características do trabalho a ser desempenho por estes profissionais.

Em síntese, importa destacar a relevância de estudos com estas características, já que possibilitam compreender que conhecimentos e competências tendem a ser mais valorizadas nos currículos do Ensino Superior. Os resultados obtidos permitem, assim, definir qual o perfil esperado dos futuros graduados, e num posterior desenvolvimento deste estudo, contrapor com o perfil profissional esperado pelo mercado de trabalho, avaliando o grau de (mis)match entre estes dois sistemas.

\section{Referencias}

Braun, V., \& Clarke, V. (2006). Using thematic analysis in psychology. Qualitative Research in Psychology, $3(2)$, 77-101. http://doi.org/10.1191/1478088706qp063oa

Dias, D., \& Amaral, A. (2014). Assessment of Higher Education learning outcomes (AHELO): Feasibility Study. In M. J. Rosa \& A. Amaral (Eds.), Quality Assurance in Higher Education. Contemporary Debates. (pp. 66-86). New York: Palgrave Macmillan. http://doi.org/10.1007/978-94-6091-867-4_8

Dias, D., \& Soares, D. (2017). Learning outcomes in Higher Education: Designing a conceptual map for portuguese academia. In INTED2017 (pp. 91889194). Valencia: IATED. http://doi.org/10.21125/inted.2017.2170

Egan, A., Maguire, R., Christophers, L., \& Rooney, B. (2017). Developing creativity in higher education for 21st century learners: A protocol for a scoping review. International Journal of Educational Research, 82, 21-27. http://doi.org/10.1016/j.ijer.2016.12.004

Gallavara, G., Hreinsson, E., Kajaste, M., \& Zadeh, M. S. (2008). Learning outcomes: Common framework different approaches to evaluation learning outcomes in the Nordic countries. Helsinki.

OPP. (2011). Código deontológico. Lisboa.

Soares, D., Franco, A., \& Dias, D. (2017). Translating critical thinking skills to higher education practices. In Proceedings of INTED2017 Conference. Valencia. http://doi.org/10.21125/inted.2017.2171

Tissot, P. (2008). Terminology of education and training policy: A multilingual glossary. (CEDEFOP, Ed.). Luxembourg.

Tremblay, K., Lalancette, D., Roseveare, D., Dias, D., \& Amaral, A. (2013). Assessment of Higher Education learning outcomes (AHELO): Feasibility Study. Feasibility Study Report, 1, 66-86. http://doi.org/10.1007/978-94-6091-867-4_8

Veiga, A., Rosa, M. J., Dias, D., \& Amaral, A. (2013). Why is it difficult to grasp the impacts of the Portuguese quality assurance system? European Journal of Education, 48(3), 454-470. http://doi.org/10.1111/ejed.12040

\section{Agradecimientos}

Este projeto de investigação tem o apoio de Agência de Avaliação e Acreditação do Ensino Superior (A3ES). 